\title{
Revascularization for Unprotected Left Main Coronary Artery Disease: An Evolution in Clinical Decision Making
}

\author{
David E. Kandzari • John A. Ormiston
}

Published online: 5 July 2011

(C) The Author(s) 2011. This article is published with open access at Springerlink.com

\begin{abstract}
Coronary artery bypass grafting (CABG) has been considered the standard therapy for unprotected (nonrevascularized) left main coronary disease (ULM). However, increasing experience with ULM percutaneous coronary intervention (PCI) has resulted in high procedural success and favorable early and late clinical outcomes. In particular, reduction in clinical restenosis with drug-eluting stents, evolution of procedural technique, and demonstration of favorable outcomes from comparative trials with CABG have promoted consideration of PCI as an alternative revascularization strategy in selected patients with ULM disease. This review summarizes the results from comparative studies examining PCI versus CABG for ULM disease, discusses changing indications for ULM PCI and identifies outstanding issues that must be considered before further advancing treatment recommendations.
\end{abstract}

Keywords Left main coronary artery. Stents · Bypass surgery · Drug-eluting stents · Guidelines · SYNTAX trial . Revascularization

D. E. Kandzari $(\bowtie)$

Piedmont Heart Institute,

Suite 300, 275 Collier Road,

Atlanta, GA 30309, USA

e-mail: david.kandzari@piedmont.org

J. A. Ormiston

Mercy Hospital,

Auckland, New Zealand
Clinical Trial Acronyms

ASAN-MAIN

ASAN Medical Center-Left MAIN

Revascularization

EXCEL

Evaluation of XIENCE PRIME

Versus Coronary Artery Bypass

Surgery for Effectiveness of Left

Main Revascularization

ISAR-LEFT MAIN Intracoronary Stenting and Angiographic Results: Drug-Eluting Stents for Unprotected Coronary Left Main Lesions

MAIN-COMPARE Revascularization for Unprotected Left Main Coronary Artery Stenosis: Comparison of Percutaneous Coronary Angioplasty Versus Surgical Revascularization from Multicenter Registry

SYNTAX Synergy Between Percutaneous Coronary Intervention with TAXUS and Cardiac Surgery

\section{Introduction}

In a lesion subset once routinely excluded from interventional cardiology trials, recent successes principally in nonrandomized comparative trials of drug-eluting stents (DES) and coronary artery bypass grafting (CABG) in unprotected left main coronary disease (ULM) revascu- 
larization have supported the rationale to revisit established conventions for treatment and broaden therapeutic options. Although varied in trial design, methods, and study population size, these trials suggest clinical equipoise for percutaneous coronary intervention (PCI) and $\mathrm{CABG}$ with consistently similar rates of combined safety outcomes of death, myocardial infarction, and stroke [1••]. In selected instances, new insights from recent studies have identified patient characteristics for which PCI may represent an acceptable alternative or possibly even preferred strategy. Accordingly, societal guidelines for ULM PCI have recently been revised. For example, the 2009 United States focused guidelines for PCI support consideration of ULM stenting in patients with anatomic conditions that are associated with a low risk of PCI-related procedural complications and clinical conditions that predict an increased risk of adverse surgical outcomes (class IIb) [2••]. Similarly, the 2010 European societal guidelines have advanced the indication for ULM PCI to either a IIa or IIb recommendation depending upon the extent and complexity of both left main and non-left main coronary disease [3••]. Considering the increasing focus on ULM PCI in clinical practice, the purpose of this document is to review the strengths and deficiencies of existing evidence that may support (or dismiss) ULM PCI, provide guidance regarding clinical decision making and address outstanding concerns that must be satisfied before further redefining standards for ULM revascularization.

\section{Contemporary Trials in ULM Percutaneous Revascularization}

Until recently, only modest evidence was available to support ULM PCI in patients ineligible for CABG with even less evidence to endorse PCI as a routine therapy for a broader patient population. Early discouraging reports with balloon angioplasty or bare metal stents (BMS) were confounded by poor patient selection and nascent procedural technique, contributing to at best inconsistent intermediate-term clinical outcomes and at worst unacceptably high rates of restenosis-related complications manifest as repeat revascularization, myocardial infarction, or even sudden cardiac death. However, encouraged by small, randomized experience and comparisons from observational trials or subgroup analysis, more contemporary studies evaluating DES for ULM disease and with longer-term follow-up have demonstrated remarkably consistent and favorable "hard" end points of death, myocardial infarction, and repeat revascularization $[1 \bullet \bullet, 4,5]$.

Outcomes of DES and BMS in ULM lesions parallel those in less complex lesion subsets, with significant reductions in restenosis and repeat revascularization and, at least similar safety outcomes of death, myocardial infarction, and stent thrombosis. In a recent meta-analysis of trials comparing BMS and DES in ULM revascularization $(N=5,081)$, treatment with DES was associated with significant reduction in the 3 -year rates of death (odds ratio [OR], 0.70; 95\% CI, 0.53-0.92; $P=0.01)$, myocardial infarction (OR, 0.49; 95\% CI, 0.26-0.92; $P=0.03$ ), and repeat revascularization (OR, 0.46; 95\% CI, 0.30-0.69; $P<$ 0.01) [6]. Although treatment with DES is preferred, outcomes with conventional BMS are important because their use (or CABG) may be favored over DES if there is increased bleeding risk, impending noncardiac surgery that requires discontinuation of dual antiplatelet therapy, and treatment of large diameter vessels $(\geq 5 \mathrm{~mm})$ that exceed DES diameter availability. Although outcomes may have been influenced by selection and treatment bias, recent nonrandomized comparison of BMS and CABG for ULM revascularization in the ASAN-MAIN registry reported lower unadjusted long-term rates of death and the composite of death, Q-wave myocardial infarction, and stroke with BMS compared with CABG (CABG; $15.9 \%$ vs $24.1 \% ; P=0.02$ and $25.2 \%$ vs $32.1 \%$; $P=0.04$, respectively); however, rate of target vessel revascularization was significantly higher in the BMS cohort $(36.7 \%$ vs $4.9 \% ; P<0.001)$ [7].

Recent trials and pooled analysis have provided important perspective to the safety and efficacy of ULM revascularization with DES compared with CABG. The nonrandomized MAIN COMPARE trial involving 2,240 patients with ULM disease compared outcomes with PCI (DES 71\%/BMS 29\%) or CABG [8•]. Notably there was less diabetes or multivessel coronary disease in the PCI cohort. At 5-year follow-up, according to propensity score adjustment including 542 matched patient pairs, ULM PCI was associated with similar mortality (hazard ratio [HR], 1.02 ; 95\% CI, 0.74-1.39) and the composite outcome of death, Q-wave myocardial infarction, and stroke (HR, 1.10; 95\% CI, 0.74-1.38) [9]. However, repeat revascularization was significantly more common with PCI compared with CABG (HR, 4.55; 95\% CI, 2.88-7.20). Analysis following risk adjustment but limited to patients treated with $\mathrm{CABG}$ or DES (396 patient pairs) resulted in similar outcomes. These data represent an important contribution to our understanding of ULM PCI but must be cautiously interpreted given that propensity adjustment in nonrandomized trials may not fully correct for differences in patient populations.

In the randomized SYNTAX trial comparing CABG with PCI for left main/multivessel disease, patient treatment assignment was stratified according to presence of significant ULM disease. Of the ULM cohort $(N=705)$, there were approximately $60 \%$ with bifurcation disease and $13 \%$ with isolated left main disease [10•]. In the ULM subgroup, despite significantly higher repeat revascularization for the 
PCI group at 3 years $(20.0 \%$ PCI vs $11.7 \%$ CABG; $P=0.004)$, and lower incidence of stroke in the PCI group (1.2\% PCI vs $4.0 \%$ CABG; $P=0.02)$, outcomes of death (7.3\% PCI vs $8.4 \%$ CABG; $P=0.64$ ), myocardial infarction (6.9\% PCI vs $4.1 \%$ CABG; $P=0.14$ ), and the composite end point of death/myocardial infarction/stroke $(13.0 \% \mathrm{PCI}$ vs $14.3 \%$ CABG; $P=0.60$ ) remained similar between treatment groups $[11 \cdot, 12]$. These results are consistent with those from a recent systematic overview of comparative trials in ULM revascularization $(N=3,773)$ reporting similar safety outcomes between percutaneous and surgical revascularization strategies but a higher rate of repeat revascularization with PCI [13].

Regarding selection of DES, few comparative studies have evaluated outcomes relative to DES type. In the randomized ISAR-LEFT MAIN trial [14•], PCI with either sirolimuseluting stents (SES) or paclitaxel-eluting stents (PES) was associated with similar 2-year clinical events in both stent groups (target lesion revascularization, 9.2\% PES vs $10.7 \%$ SES; $P=0.47$ ). Although to date no studies have compared newer-generation DES to PES or SES in ULM PCI, ongoing trials are comparing newer-generation DES to CABG. The Nordic-Baltic-British Left Main Revascularization trial is randomizing 1,200 patients with ULM and SYNTAX score less than 22 to CABG or DES with end points of major adverse cardiac events (MACE) at 2 years and death at 5 years. The EXCEL trial is a large, international trial in which approximately 3,000 patients with ULM disease and a SYNTAX score $\leq 32$ will be randomized to revascularization with everolimus-eluting stents or CABG.

\section{Patient Selection and Predictive Models for Outcomes Assessment Following ULM Revascularization}

Although not all uncertainties must be satisfied to extend ULM PCI as an alternative to surgery in broad patient populations, presently available data do permit advancing ULM PCI in more narrowly defined subgroups of patients. However, translation of clinical data into results meaningful to an individual patient is challenging, and the presentation of clinical data to patients may be biased by preferential selection of clinical studies and differential emphasis on end points (eg, death vs repeat revascularization vs recovery and quality of life). Specific to ULM revascularization, for which a surgical standard of care has been historically established, and in which both PCI and surgical alternatives may result in divergent clinical outcomes favoring either therapy depending upon risk, a predictive model based on clinical and angiographic characteristics seems essential to clinical decision making and informing patients for consent.

The overall extent and complexity of both left main and non-left main coronary disease are important for deciding revascularization strategy. Although anatomic location of disease within the left main segment has been identified as an important predictor of clinical outcome following PCI (detailed below), the extent, severity, and complexity of non-left main disease may be an even greater determinant of risk. Specifically, non-left main lesion complexity (eg, chronic total occlusion, bifurcation disease, calcification) may jeopardize the likelihood of procedural success and limit completeness of revascularization, which was significantly less common among PCI than CABG patients in the ULM subgroup of the SYNTAX trial [10 ${ }^{\bullet}$. Among patients undergoing ULM PCI, the extent of coronary disease also predicts likelihood of late-term major adverse events, principally driven by increasing risk of repeat revascularization $[11 \bullet, 12]$.

Risk scores are useful in determining the early and late outcomes after PCI and CABG for ULM disease, and discriminating between these two modalities for the individual patient [15•]. The EuroSCORE and Parsonnet score, which are typically used to risk stratify CABG candidates, have been applied both prospectively and retrospectively to patients undergoing ULM PCI. An analysis from the MAINCOMPARE trial demonstrated that the EuroSCORE $\geq 6$ was an independent predictor of mortality in ULM patients who undergo both percutaneous and surgical revascularization [16]. Similarly, increasing Parsonnet score was also identified as a significant predictor of major adverse cardiac and cerebrovascular events [16].

Predictive models, such as the SYNTAX score, may also help discussions of risk and benefit during the consent process for ULM revascularization $[11 \bullet, 17]$. When the SYNTAX score is in the highest tercile $(\geq 33)$ indicating extensive and/or complex coronary artery disease, surgical revascularization may be favored over PCI. Alternatively, patients with low (0-22) and intermediate (23-32) SYNTAX scores may have comparable composite safety and efficacy outcomes with either treatment strategy [11•]. At 3 years, in patients with ULM disease and SYNTAX scores $\geq 33$, PCI patients had higher rates of repeat revascularization than $\mathrm{CABG}(27.7 \%$ vs $9.2 \% ; P<0.001)$ and death $(13.4 \%$ vs $7.6 \% ; P=0.10)$ [12]. Conversely, patients with ULM with low (0-22) and intermediate (2332) SYNTAX scores had similar rates of repeat revascularization, myocardial infarction, death, or stroke or any combination of these if treated by PCI or CABG. Although these analyses from the left main cohort of the SYNTAX trial are post hoc and statistically underpowered, they currently represent the best level of evidence to guide revascularization decisions, and have thus recently been incorporated into societal guideline recommendations. As an example, the 2010 European Society of Cardiology/ European Association for Cardio-Thoracic Surgery guidelines for myocardial revascularization have revised the 
indication for ULM PCI involving the ostium/shaft with or without single vessel coronary disease to a class IIa recommendation (weight of evidence favors its efficacy) [3••]. ULM PCI for ostial/shaft disease with two- or threevessel disease, or distal bifurcation disease of the left main with two- or three-vessel disease and a SYNTAX score less than 33 is provided a class IIb recommendation (usefulness is less well established). Alternatively, a class III recommendation for PCI is applied for ULM patients with a SYNTAX score $\geq 33$ (evidence that treatment is not useful and may be harmful).

For any risk model, prospective validation in an independent patient population with ULM disease is essential. Observational studies have externally validated the utility of the SYNTAX score to predict mortality and major adverse cardiac events in usual practice $[18,19]$. In addition to the SYNTAX score, the incorporation of clinical risk factors besides angiographic characteristics may improve the predictive utility. The New Risk Stratification (NERS) model includes clinical, procedural, and angiographic characteristics [20]. For MACE, the sensitivity and specificity of an NERS score $\geq 25$ were $92.0 \%$ and $74.1 \%$, respectively, representing significantly higher predictive measures than SYNTAX intermediate risk $(20.5 \%$ and $25.4 \%)$ or SYNTAX higher risk scores $(70.5 \%$ and $35.2 \% ; P<0.001$ for all comparisons). Furthermore, an NERS score $\geq 25$ was the only independent predictor of MACE and stent thrombosis.

Currently, no uniform consensus exists regarding the most practical and accurate risk model for evaluating patients with ULM disease. One additional challenge is that patients considered for ULM PCI have been systematically excluded from many clinical trials, further restricting the generalizability of data to any individual patient. Acknowledging the limitation that risk scores cannot predict outcomes for individual patients with characteristics not included in the model, construction of a risk model must include appropriate and necessary preprocedural clinical and angiographic characteristics while avoiding overfitting the model [15•].

Finally, excepting emergency indications, ad hoc PCI should not be performed. Instead, the process for patient selection and informed consent ideally should involve a collaborative, multidisciplinary approach with a "Heart Team" represented by both a cardiac surgeon and interventional cardiologist, with both having the opportunity to discuss with the patient the relative merits and risk of each strategy $[3 \bullet \bullet$. Consultation with a non-interventional cardiologist may also provide the patient more insight into the treatment options. Recognizing the informed consent process as an opportunity to optimize patient understanding and objective decision making, the Heart Team represents a balanced and integrated approach for multidisciplinary decision making and consensus building.

\section{Special Considerations for ULM Percutaneous Revascularization}

\section{Left Main Lesion Complexity}

Left main coronary lesion complexity has clear procedural and clinical implications underscoring the need for proper evaluation of both the distribution and severity of disease. To this purpose, hemodynamic and intravascular ultrasound (IVUS) assessment of left main coronary atherosclerotic disease has associated the functional and/or anatomic relevance of stenosis with the need for treatment and clinical outcome [21, 22]. Specifically, an IVUS-derived minimal luminal area of less than $6.0 \mathrm{~mm}^{2}$ has been found to be a useful cutoff value for clinically significant left main coronary artery disease [23], and has been correlated with hemodynamic significance by fractional flow reserve assessment [22]. Recent IVUS studies have demonstrated that left main atherosclerotic plaque burden is frequently more extensive than predicted by angiography alone [24, 25]; in particular, whereas atherosclerotic plaque is rarely present in the carina (flow divider), extension of disease from the ostia of the left anterior descending artery or left circumflex arteries into the left main segment is very common.

Left main lesions not involving the distal bifurcation (representing $<40 \%$ of patients undergoing revascularization) are associated with high procedural success rates and favorable late-term outcomes of death, myocardial infarction, and repeat revascularization. In a multicenter study of 147 patients undergoing ostial or shaft ULM PCI with DES, rates of cardiac death rate and repeat target lesion revascularization were $2.7 \%$ and $0.7 \%$, respectively, during an average followup period of approximately 2.5 years [26]. In comparison, the presence of distal bifurcation disease has repeatedly been identified as one of the most significant predictors of repeat revascularization and overall MACE after ULM PCI. A meta-analysis of 17 trials involving ULM PCI identified the presence of bifurcation disease as the most significant predictor of repeat revascularization and overall MACE [27].

\section{Procedural Technique}

Percutaneous coronary intervention for ULM disease can be technically challenging, requiring optimal strategies for treatment of either complex distal bifurcation disease or ostial/shaft stenoses that jeopardize a large myocardial territory. Somewhat surprisingly, however, procedural strategy and technique are common practical considerations that are poorly addressed in clinical trials describing left main revascularization. In part related to this reason, optimal PCI strategies for ULM disease are yet to be clearly defined. Moreover, strategies may vary depending on different anatomic features of the entire coronary anatomy and lesion morphology. 
Treatment of distal bifurcation lesions is technically more challenging and is associated with higher rates of restenosis than isolated ostial or shaft disease, particularly when two stents are used. When angiographic restenosis does occur, it is most common at the ostium of the left circumflex artery [28], a clinical observation that may be dependent upon bifurcation angle and carina shift rather than change in plaque geometry. In accord with published results involving bifurcation stenting in non-left main disease, uncontrolled studies of ULM bifurcation PCI favor a single-stent provisional approach compared with intentional two-stent techniques. Notably, one large study reported comparatively higher rates of cardiovascular death and target lesion revascularization with two-stent treatment of bifurcation disease, including instances of unsuccessful provisional approaches that required additional stent placement [28]. In addition, several recent observational studies have demonstrated nearly equivalent clinical outcomes with single stent ULM bifurcation revascularization and left main stenting for ostial or shaft disease [29-32]. Nevertheless, approximately $40 \%$ of ULM bifurcation treatment involves a two-stent method [14•, 28], yet the optimal twostent technique (eg, crush, culotte, V- or T-stenting) has not been identified, and the procedure is instead determined more by operator and institutional preference.

Intended as a solution to limitations of existing stent designs in complex anatomy, novel dedicated bifurcation stent designs are in early clinical development [33], yet evidence to support their procedural and clinical superiority over existing standards has not been sufficiently demonstrated.

Aside from stent technique, additional procedural uncertainties relate to use of IVUS and hemodynamic support. In many circumstances, the application of IVUS may be invaluable to assess optimally plaque distribution, bifurcation involvement, and vessel calcification that are characteristics often poorly defined by angiography alone. IVUS may also provide important information regarding stent sizing, post deployment stent expansion, and stent-wall apposition. Nevertheless, the application of IVUS in ULM PCI trials has been inconsistent, with some studies reporting improved survival with IVUSguided ULM PCI [34] and others describing favorable outcomes despite negligible use of IVUS [14•].

Similarly, patient-specific angiographic- and proceduralrelated factors that predict the unplanned requirement for adjunctive hemodynamic support during ULM PCI are poorly characterized. In most instances, however, pharmacologic or mechanical circulatory support is not required; in the ISAR-LEFT MAIN trial, for example, intra-aortic balloon pump counterpulsation was used in less than $1 \%$ of the 607 patients undergoing ULM PCI [14•]. Although procedural-related complications [35] or hemodynamic compromise [36] may be reduced with elective use of intra-aortic balloon pump counterpulsation or alternative methods of hemodynamic support [37], a reduction in adverse clinical outcomes (eg, myocardial infarction, death) compared with their provisional use has not been demonstrated.

\section{Antiplatelet Therapy and Stent Thrombosis}

DES placement in ULM disease represents a dilemma between the importance of avoiding restenosis and the risk of stent thrombosis associated with delayed vessel healing. Given that stent thrombosis is a devastating complication of PCI, associated with a near uniform rate of myocardial infarction and considerable mortality [38], its occurrence in the ULM territory may have catastrophic clinical consequences. Fortunately, ULM stent thrombosis is uncommon, and several recent multicenter registries evaluating the occurrence of late and very late stent thrombosis provide some reassuring and remarkably consistent evidence to support DES treatment in ULM disease. In the ISAR-LEFT MAIN trial, for example, among 607 patients receiving DES for ULM disease, the overall 2-year rate of Academic Research Consortiumdefined definite stent thrombosis was $0.5 \%$, with no instances beyond 30 days of the index procedure [14•].

Thienopyridine discontinuation within 6 months of DES implantation is a predictor of stent thrombosis [38]. In a multicenter observational study, clopidogrel discontinuation within the first 31 to 180 days following ULM DES implantation was associated with a more than fourfold riskadjusted increase in cardiovascular mortality and myocardial infarction compared with discontinuation beyond 180 days [39]. Alternatively, with dual antiplatelet therapy extended beyond 1 year, combined data from two randomized trials with DES recently reported no significant reduction in cardiovascular death, myocardial infarction, or stent thrombosis [40] but only $3 \%$ of patients included in this analysis underwent ULM PCI.

In ULM PCI clinical trials, the duration of antiplatelet therapy has been variable, with no standardized recommendations for aspirin and thienopyridine dosing or duration. In addition, limited studies have focused on the role of genomic and/or platelet reactivity testing to identify patients with high on-treatment residual platelet activity who may be at increased risk for subsequent ischemic events [41]. Accordingly, the optimal duration of dual antiplatelet therapy in patients stented for ULM disease has not been established. Thus, expectations for patient compliance and a reasonable assurance of no foreseeable circumstances that might necessitate premature discontinuation of antiplatelet therapy are important considerations. $\mathrm{CABG}$ or even BMS should be considered if concerns are present regarding bleeding risk, noncompliance, foreseeable need for interruption, or contraindications to prolonged dual antiplatelet therapy. 
Angiographic and Clinical Surveillance

Historically, routine surveillance angiography was common, but considering the low rates of DES restenosis and stent thrombosis following ULM revascularization, the clinical utility of surveillance of angiography has been challenged. In the LE MANS substudy $(N=145)$ of the SYNTAX trial, for example, angiographic restenosis ( $>50 \%$ stenosis) at 15 -month follow-up was identified in only $2 \%(1 / 48)$ of patients with ostial/shaft disease and $10 \%$ (10/97) of patients with bifurcation disease [42]. In consideration of these data and the recognition that scheduled nonsymptomdriven angiography could result in unnecessary procedures, the most recent guidelines no longer endorse its performance $[2 \bullet \bullet$. However, noninvasive assessment of ischemia is reasonable at 6 months and annually thereafter $[1 \bullet \bullet]$.

When clinical restenosis following ULM stenting is identified, the most appropriate treatment is also uncertain. In cases of intermediate significance, assessment of hemodynamic significance with fractional flow reserve should be performed. If lesion significance is confirmed, IVUS may also inform the mechanism of restenosis and should be performed routinely for restenosis if repeat PCI is considered.

For patients with ULM restenosis, repeat revascularization seems imperative, and PCI may be associated with favorable outcomes. In a multicenter observational study of patients with ULM restenosis treated with repeat PCI $(N=70)$, the risk of cardiovascular death was $1.7 \%$ over a mean follow-up period of 35 months, with no occurrences of stent thrombosis [43]. During the follow-up period, the risk of MACE was lowest with CABG or repeat PCI compared with medical therapy alone (MACE at 35 months, 14\% CABG, $25 \%$ PCI, $50 \%$ medical therapy). Unlike after DES treatment of de novo ULM disease, early follow-up angiography at 4 to 6 months should be considered if repeat PCI is performed for ULM restenosis given higher risk of disease recurrence.

\section{Conclusions}

Over the past 5 years, an evidence base has emerged supporting the consideration of ULM PCI as an alternative revascularization strategy to $\mathrm{CABG}$ in selected patients. In particular, trials reporting late-term safety outcomes of cardiovascular death, myocardial infarction, and stroke comparable to $\mathrm{CABG}$ have fostered renewed enthusiasm for ULM PCI. In parallel, application of DES and advances in technique and strategy have enhanced the rates of early procedural and long-term clinical success of ULM PCI.

Ultimately, demonstration of at least clinical equivalence in a randomized trial comparing ULM PCI with surgery is necessary before percutaneous revascularization can be routinely accepted as an alternative to bypass surgery; to this purpose, forthcoming trials designed with careful attention to patient selection, timing of end-point ascertainment, and relevance of safety and efficacy end points should inform clinical decision making and treatment guidelines. However, consistent with many large comparative trials, it is more likely that such studies may clarify which patients with ULM are suitable for both or either therapies rather than demonstrate clinical equivalence for a broadly defined patient population with individual characteristics that pose variable risk.

Presently available data are substantive enough to support ULM PCI in the absence of coexisting complex coronary disease (eg, SYNTAX score $\geq 33$ ). Thus, an important focus should be the responsibility of appropriate patient selection, with consensus that represents full consultation ideally with both an interventionalist and cardiac surgeon. Integration of clinical and angiographic variables into risk models may further refine assessment of patient risk for either revascularization method and responsibly inform the patient for consent. Aside from an emphasis on comparative clinical outcomes, ongoing clinical trials intended to address the practical and technique-related issues of ULM PCI and promote consensus building between cardiology and surgical societies are essential to advance treatment recommendations and optimize the outcomes for patients with ULM coronary artery disease.

Disclosure Conflicts of interest: D.E. Kandzari: has received research/grant support from Abbott Vascular, Medtronic, and Cordis Corp.; and has received consulting honoraria from Abbott Vascular, Medtronic, and Micell Technologies; J.A. Ormiston: serves on the board for Abbott Vascular and Boston Scientific, and receives honoraria from Abbott Vascular and Boston Scientific.

Open Access This article is distributed under the terms of the Creative Commons Attribution Noncommercial License which permits any noncommercial use, distribution, and reproduction in any medium, provided the original author(s) and source are credited.

\section{References}

Papers of particular interest, published recently, have been highlighted as:

- Of importance

•- Of major importance

1. • Kandzari DE, Colombo A, Park SJ, et al. Revascularization for unprotected left main disease: evolution of the evidence basis to redefine treatment standards. J Am Coll Cardiol. 2009;54:157688. This position paper from the American College of Cardiology (ACC) Interventional Council summarizes the practical and clinical trial issues with ULM PCI. The manuscript also provides a comprehensive summary of comparative ULM PCI versus CABG trials.

2. • Kushner FG, Hand M, Smith SC, et al. 2009 focused updates: ACC/AHA guidelines for the management of patients with ST- 
Elevation myocardial infarction (updating the 2004 guideline and 2007 focused update) and ACC/AHA/SCAI guidelines on percutaneous coronary intervention (updating the 2005 guideline and 2007 focused update): a report of the American College of Cardiology Foundation/American Heart Association Task Force on Practice Guidelines. Circulation 2008;117:261-95. This revision of the ACC/American Heart Association guidelines advanced the performance of ULM PCI from a class III to class IIb recommendation. In addition, routine angiographic surveillance following ULM PCI is no longer recommended.

3. • Wijns W, Kolh P, Danchin N, et al. Guidelines on myocardial revascularization: The Task Force on Myocardial Revascularization of the European Society of Cardiology (ESC) and the European Association for Cardio-Thoracic Surgery (EACTS). Developed with the special contribution of the European Association for Percutaneous Cardiovascular Interventions (EAPCI). Eur Heart J. 2010 Aug 29. [Epub ahead of print]. This revision of the ESC guidelines advanced the performance of ULM PCI to class IIa or IIb recommendation, depending upon lesion complexity. In addition, the guidelines place emphasis on the role of the SYNTAX score and the collaboration between interventionalists and surgeons (Heart Team) in clinical decision making.

4. Park SJ, Park DW. Percutaneous coronary intervention with stent implantation versus coronary artery bypass surgery for treatment of left main coronary artery disease. Is it time to change guidelines? Circ Cardiovasc Intervent. 2009;2:59-68.

5. Boudriot E, Thiele H, Walther T, et al. Randomized comparison of percutaneous coronary intervention with sirolimus-eluting stents versus coronary artery bypass grafting in unprotected left main stem stenosis. J Am Coll Cardiol. 2011;57:538-45.

6. Pandya SB, Kim YH, Meyers SN, et al. Drug-eluting versus bare-metal stents in unprotected left main coronary artery stenosis: a meta-analysis. J Am Coll Cardiol Cardiovas Interv. 2010;3:602-11.

7. Park D-W, Kim Y-H, Yun S-C, et al. Long-term outcomes after stenting versus coronary artery bypass grafting for unprotected left main coronary artery disease: 10 -year results of bare-metal stents and 5-year results of drug-eluting stents from the ASAN-MAIN (ASAN Medical Center-Left MAIN Revascularization) registry. J Am Coll Cardiol. 2010;56:1366-75.

8. - Seung KB, Park DW, Kim YH, et al. Stents versus coronaryartery bypass grafting for left main coronary artery disease. $\mathrm{N}$ Engl J Med. 2008;358:1781-92. The MAIN-COMPARE trial is one of the largest (nonrandomized) comparative trials of ULM $P C I$ versus $C A B G$, demonstrating similar early and late-term death, myocardial infarction, and stroke between the two revascularization strategies, despite more common repeat revascularization with PCI.

9. Park DW, Seung KB, Kim YK, et al. Long-term safety and efficacy of stenting versus coronary artery bypass grafting for unprotected left main coronary artery disease: 5 -year results from the MAIN-COMPARE (Revascularization for Unprotected Left Main Coronary Artery Stenosis: Comparison of Percutaneous Coronary Angioplasty Versus Surgical Revascularization) Registry. J Am Coll Cardiol. 2010;56:117-24.

10. - Serruys PW, Morice MC, Kappetein AP, et al. Percutaneous coronary interventions versus coronary-artery bypass grafting for severe coronary artery disease. N Engl J Med. 2009;360:961-72. The SYNTAX trial represents the largest and most contemporary randomized comparative trials of PCI versus $C A B G$ for left main and multivessel coronary disease; at 1 year, the study demonstrated similar rates of the composite of death, myocardial infarction, and stroke between the two revascularization strategies, despite more common repeat revascularization with $P C I$.

11. - Morice MC, Serruys PW, Kappetein AP, et al. Outcomes in patients with de novo left main disease treated with either percutaneous coronary intervention using paclitaxel-eluting stents or coronary artery bypass graft treatment in the Synergy Between Percutaneous Coronary Intervention With TAXUS and Cardiac Surgery (SYNTAX) Trial. Circulation 2010;121:2645-53. Among patients with left main disease in the SYNTAX trial, this report from the trial substudy describes comparable outcomes with ULM PCI compared with CABG for patients with low and intermediate SYNTAX scores.

12. Serruys P. Three-year outcomes for unprotected left main subgroup in the SYNTAX Trial. Presented at Transcatheter Therapeutics 2010 Scientific Sessions. Washington, DC; September 25, 2010.

13. Naik H, White AJ, Chakravarty T, et al. A meta-analysis of 3,773 patients treated with percutaneous coronary intervention or surgery for unprotected left main coronary artery stenosis. J Am Coll Cardiol Intv. 2009;2:739-47.

14. - Mehilli J, Kastrati A, Byrne R, et al. for the ISAR-LEFT MAIN (Intracoronary Stenting and Angiographic Results: Drug-Eluting Stents for Unprotected Coronary Left Main Lesions) Study Investigators. Paclitaxel- versus sirolimus-eluting stents for unprotected left main coronary artery disease. J Am Coll Cardiol. 2009;53:1760-8. The ISAR-LEFT MAIN trial was a large, singlecenter, randomized trial comparing percutaneous revascularization with sirolimus- or paclitaxel-eluting stents for ULM disease. The trial demonstrated similar safety and efficacy between both $D E S$, in particular favorably low rates of stent thrombosis and clinical restenosis.

15. • Garg S, Stone GW, Kappetein AP, Sabik JF, Simonton C, Serruys PW. Clinical and angiographic risk assessment in patients with left main stem lesions. J Am Coll Cardiol Intv. 2010;3:891-901. This summary document provides an overview of risk score models that may be considered in revascularization decision making for ULM disease.

16. Min SY, Park DW, Yun SC, et al. Major predictors of long-term clinical outcomes after coronary revascularization in patients with unprotected left main coronary disease: analysis from the MAINCOMPARE study. Circ Cardiovasc Interv. 2010;3:127-33.

17. Sianos G, Morel MA, Kappetein AP, et al. The SYNTAX score: an angiographic tool grading the complexity of coronary artery disease. EuroIntervention. 2005;1:219-27.

18. Capodanno D, Di Salvo ME, Cincotta G, Miano M, Tamburino C, Tamburino C. Usefulness of the SYNTAX score for predicting clinical outcome after percutaneous coronary intervention of unprotected left main coronary artery disease. Circ Cardiovasc Interv. 2009;2:302-8.

19. Kim YH, Park DW, Kim WJ, et al. Validation of SYNTAX (Synergy between PCI with Taxus and Cardiac Surgery) score for prediction of outcomes after unprotected left main coronary revascularization. J Am Coll Cardiol Intv. 2010;3:612-23.

20. Chen SL, Chen JP, Mintz G, et al. Comparison between the NERS (New Risk Stratification) score and the SYNTAX (Synergy Between Percutaneous Coronary Intervention with Taxus and Cardiac Surgery) score in outcome prediction for unprotected left main stenting. J Am Coll Cardiol Intv. 2010;3:632-41.

21. Bech GJW, Droste H, Pijls NH, et al. Value of fractional flow reserve in making decisions about bypass surgery for equivocal left main coronary artery disease. Heart. 2001;86:547-52.

22. Jasti V, Ivan E, Yalamanchili V, Wongpraparut N, Leesar MA. Correlations between fractional flow reserve and intravascular ultrasound in patients with an ambiguous left main coronary artery stenosis. Circulation. 2004;110:2831-6.

23. Abizaid AS, Mintz GS, Abizaid A, et al. One-year follow-up after intravascular ultrasound assessment of moderate left main coronary artery disease in patients with ambiguous angiograms. J Am Coll Cardiol. 1999;34:707-15. 
24. Tamburino C, Capranzano P, Capodanno D, et al. Plaque distribution patterns in distal left main coronary artery to predict outcomes after stent implantation. J Am Coll Cardiol Intv. 2010;3:624-31.

25. Oviedo C, Maehara A, Mintz GS, et al. Intravascular ultrasound classification of plaque distribution in left main coronary artery bifurcations: where is the plaque really located? Circ Cardiovasc Interv. 2010;3:105-12.

26. Chieffo A, Park SJ, Valgimigli M, et al. Favorable long-term outcome after drug-eluting stent implantation in nonbifurcation lesions that involve unprotected left main coronary artery: a multicenter registry. Circulation. 2007;116:158-62.

27. Biondi-Zoccai GG, Lotrionte M, Moretti C, et al. A collaborative systematic review and meta-analysis on 1278 patients undergoing percutaneous drug-eluting stenting for unprotected left main coronary artery disease. Am Heart J. 2008;155:274-83.

28. Palmerini T, Marzocchi A, Tamburino $\mathrm{C}$, et al. Impact of bifurcation technique on 2-year clinical outcomes in 773 patients with distal unprotected left main coronary artery stenosis treated with drug-eluting stents. Circ Cardiovasc Interv. 2008;1:185-92.

29. Park SJ, Kim YH, Lee BK, et al. Sirolimus-eluting stent implantation for unprotected left main coronary artery stenosis: comparison with bare metal stent implantation. J Am Coll Cardiol. 2005;45:351-6.

30. Kim YH, Park SW, Hong MK, et al. Comparison of simple and complex stenting techniques in the treatment of unprotected left main coronary artery bifurcation stenosis. Am J Cardiol. 2006;97:1597-601.

31. Valgimigli M, Malagutti P, Rodriguez Granillo G, et al. Singlevessel versus bifurcation stenting for the treatment of distal left main coronary artery disease in the drug-eluting stenting era: clinical and angiographic insights into the Rapamycin-Eluting Stent Evaluated at Rotterdam Cardiology Hospital (RESEARCH) and Taxus-Stent Evaluated at Rotterdam Cardiology Hospital (T-SEARCH) registries. Am Heart J. 2006;152:896-902.

32. Park SJ, Lee CW, Kim YH, Lee JH, Hong MK, Kim JJ, et al. Technical feasibility, safety, and clinical outcome of stenting of unprotected left main coronary artery bifurcation narrowing. Am J Cardiol. 2002;90:374-8.

33. Verheye S, Agostoni P, Dubois CL, et al. 9-month clinical, angiographic, and intravascular ultrasound results of a prospective evaluation of the Axxess self-expanding biolimus A9-eluting stent in coronary bifurcation lesions: the DIVERGE (Drug-Eluting Stent Intervention for Treating Side Branches Effectively) study. J Am Coll Cardiol. 2009;53:1031-9.

34. Park SJ, Kim YH, Park DW, et al. Impact of intravascular ultrasound guidance on long-term mortality in stenting for unprotected left main coronary artery stenosis. Circ Cardiovasc Interv. 2009;2:167-77.

35. Perera D, Stables R, Thomas M, et al. Elective intra-aortic balloon counterpulsation during high-risk percutaneous coronary ntervention. A randomized controlled trial. JAMA. 2010;304:867-74.

36. Briguori C, Airoldi F, Chieffo A, et al. Elective versus provisional intraaortic balloon pumping in unprotected left main stenting. Am Heart J. 2006;152:565-72.

37. Cheng JM, den Uil CA, Hoeks SE, van der Ent M, Jewbali LS, van Domburg RT, et al. Percutaneous left ventricular assist devices vs. intra-aortic balloon pump counterpulsation for treatment of cardiogenic shock: a meta-analysis of controlled trials. Eur Heart J. 2009;30:2102-8.

38. Iakovou I, Schmidt T, Bonizzoni E, et al. Incidence, predictors, and outcome of thrombosis after successful implantation of drugeluting stents. JAMA. 2005;293:2126-30.

39. Palmerini T, Marzocchi A, Tamburino C, et al. Temporal pattern of ischemic events in relation to dual antiplatelet therapy in patients with unprotected left main coronary artery stenosis undergoing percutaneous coronary intervention. J Am Coll Cardiol. 2009;53:1176-81.

40. Park SJ, Park DW, Kim YH, et al. Duration of dual antiplatelet therapy after implantation of drug-eluting stents. N Engl J Med. 2010;362:1374-82.

41. Migliorini A, Valenti R, Marcucci R, et al. High residual platelet reactivity after clopidogrel loading and long-term clinical outcome after drug-eluting stenting for unprotected left main coronary disease. Circulation. 2009;120:2214-21.

42. Marie-Claude Morice. SYNTAX left main PCI treatment: technical considerations. Presented at Transcatheter Therapeutics 2008. Washington, DC; October 14, 2008.

43. Sheiban I, Sillano D, Biondi-Zoccai G, et al. Incidence and management of restenosis after treatment of unprotected left main disease with drug-eluting stents 70 restenotic cases from a cohort of 718 patients: FAILS (Failure in Left Main Study). J Am Coll Cardiol. 2009;54:1131-6. 